\title{
Confidentiality and serious harm in genetics - preserving the confidentiality of one patient and preventing harm to relatives
}

\author{
Anneke Lucassen ${ }^{*}$ and Michael Parker $^{2}$ \\ ${ }^{1}$ Department of Clinical Genetics, Level G The Princess Anne Hospital, Southampton SO16 5YA, UK; ${ }^{2}$ Oxford Genetics \\ Knowledge Park, ETHOX, Oxford, OX37LF, UK
}

Genetics can pose special challenges to the principle of confidentiality within the health professionalpatient relationship, since genetic information is by its nature both individual and familial. Most professional guidelines allow confidentiality to be broken in rare circumstances, where it will prevent a 'serious', 'imminent' and 'likely' harm. We argue that the types of harms that may result from genetic medicine are particularly diverse. Using clinical examples, we explore ethical issues that arise when balancing individual and family member interests. As genetic testing becomes more widespread, situations will arise where clinicians are faced with a choice between preserving the confidentiality of one patient and preventing harm to another. Professionals need to incorporate the notion of familial implications in their counseling of individuals. Since such dilemmas have been relatively rare in the pre-genetic age, we call for a wider debate on the balance between confidentiality and harm to others.

European Journal of Human Genetics (2004) 12, 93-97. doi:10.1038/sj.ejhg.5201118

Published online 12 November 2003

Keywords: confidentiality; disclosure; autonomy; familial; clinical genetics; predictive testing; consent

\section{Introduction}

Respect for patient confidentiality is an essential feature of good medical practice. However, it has also long been recognised that breaching confidentiality may also be a feature of good practice under certain circumstances. This may be because of concern for broader public interests, such as is the case in 'notifiable' diseases. Or, it may be where the breaching of confidentiality has the potential to protect the patient or other individuals from serious harms. But how is this to be interpreted in genetic practice? When does a harm become serious enough to justify a breach of patient confidentiality? While some guidelines do provide examples of situations in which a harm may be sufficiently serious ${ }^{1-3}$, the assessment of the seriousness of the harm in particular cases falls to the judgement of the clinician. This can leave clinicians feeling uncertain of how to interpret

*Correspondence: Dr Anneke Lucassen. Tel: + 44 (0)23 8079 4503; Fax: 44 (0)23 8079 4346; E-mail: annekel@soton.ac.uk Received 9 July 2003; revised 11 September 2003; accepted 2 October 2003 the guidelines in a particular case. In this paper, using clinical examples in which one family member does not wish to communicate the relevant genetic information to 'at-risk' relatives, we explore the factors that need to be taken into consideration in any assessment of whether it is acceptable to breach patient confidentiality in genetics. Genetics is an interesting test case for confidentiality, because of the diversity of potential harms, the wide variety of risks of occurrence of such harms (from near certainty to very low probability) and the variability of available interventions (from completely effective treatment, to surveillance and to situations in which the only option is reproductive choice). This diversity means that a model that makes such assessment easier in genetics also has the potential to be of wider relevance in medicine.

The value of respect for patient confidentiality Why is confidentiality important?

It has long been recognised that there are limits to confidentiality and that circumstances sometimes occur 
in which the right thing to do is to breach confidentiality. These exceptions only serve to highlight the fact that, in general, great value is placed on respect for confidentiality in health care. There are a number of ways in which respect for confidentiality might be justified morally. One is with respect to patient choice: It is widely accepted that competent patients should have a control over decisions concerning their own medical care. Respect for patient choice means that they should also have a control over what happens to the information shared by them with their health professional. Patients in general accept that health professionals sometimes need to discuss this information with colleagues in the interests of their care, and perhaps too in the interest of training. But this is very different to the sharing of patient information with those not involved in their clinical care. Patients reasonably expect that, unless they have given specific permission, health professionals will not disclose the information revealed during the professional relationship to others. To take patient choice and patient autonomy seriously is to place great value on confidentiality in the doctor-patient relationship.

A second type of justification for the central role of confidentiality is the beneficial consequences of a practice that respects patient confidentiality. Patients visit their doctors at least partly because they feel that they can speak freely. That is, because they trust their doctors to respect their confidentiality. Breaches in confidentiality have the potential to seriously undermine trust in the doctorpatient relationship, and hence mean that patients do not get the support and health care that they need. Without rules regarding confidentiality, patients may be reluctant to give the health professionals the information they need, or they may be reluctant to come to the clinic in the first place.

Both these arguments also provide support for the idea that it may, on occasion, be right to breach confidentiality. If the benefits of maintaining confidentiality in a particular case are outweighed by those of breaching it, such as, for example, in cases where the death of a third party can be avoided, then breaching confidentiality will be justified. Any benefits to be gained by such a breach will need to be weighed against the benefits of having a system in which confidentiality is taken seriously, and so, this means that the standard of harm in justifying breaches will be relatively high. Where failure to breach confidentiality will very seriously undermine the autonomy of third parties, for example, through their avoidable death, it will be right to breach confidentiality. But once again, the standard will be high.

\section{Guidelines on confidentiality}

These ethical justifications for the breaching of confidentiality on rare occasions are reflected in the relevant guidelines and law. A common theme to recommendations concerning disclosure is that it should only be allowable 'in exceptional circumstances'. That is, if the risk is 'serious' or 'avoidable' and 'imminent' or 'likely'. ${ }^{4}$ The legal situation in the United Kingdom (and professional guidance ${ }^{5}$ ) is that clinicians may breach their patient's confidentiality, to an appropriate body where there is a risk of death or serious harm to a third party. ${ }^{6}$ In the United States, the Tarasoff case $^{7}$ has also been taken to imply that a clinician has a duty to disclose a real threat of serious harm although there has been some debate about the correct interpretation of this and other cases since. ${ }^{4,8}$ Several bodies have considered the role of confidentiality in genetics specifically. Unfortunately, however, they have often come to contradictory conclusions and have made conflicting recommendations. Some bodies have concluded that confidentiality should always be paramount. The UK House of Commons Science and Technology Committee, for example, argued that failure to guarantee confidentiality would discourage people from seeking information that would be beneficial to their own health, and that the individual's decision to withhold information should be final. ${ }^{9}$ French legislation on confidentiality also dictates that no disclosure can be made without consent. ${ }^{10}$ Others, however, have come down in favour of a broader duty of care including other family members in addition to the patient. ${ }^{11}$ The Human Genome Organisation (HUGO) ${ }^{12}$ concluded that ' ... shared biological risks create special interests and moral obligations...that may outweigh individual wishes.' The Nuffield Council on Bioethics also concluded that, in 'exceptional' cases, health professionals might be justified in disclosing genetic information to other family members, despite an individual's desire for confidentiality. ${ }^{13}$ In the UK, the Human Genetics Commission also concludes that the disclosure of sensitive personal genetic information...... may occasionally be justified' and that this would arise where a patient refuses to consent to such disclosure and the benefit of disclosure substantially outweighs the patient's claim to confidentiality. ${ }^{1}$ While also supporting the legitimacy of disclosure in some cases, the UK Genetics Interest Group (GIG) concluded that this is only justifiable if, in addition to the seriousness of the harm, there is the realistic possibility of an effective intervention. ${ }^{3}$

\section{Risk seriousness and the availability of 'interventions'}

An assessment of whether a breach is justified in any particular case will therefore need to take into account three different but related considerations. Firstly, there will need to be an assessment of the seriousness of the harm itself. Secondly, there will need to be an assessment of the likelihood of the harm occurring. Finally, there will need to be an assessment of the availability of effective interventions, or other options. 


\section{Seriousness of the harm}

Genetic conditions present a wide variety of possible harms of differing degrees of seriousness. There are some conditions, such as long QT syndrome (LQT) in which there is the risk of sudden death, others such as familial cancer syndromes in which there is a risk of cancer. At the other end of the scale, there are conditions where the harms might be considered less serious, for example, Von Willebrand disease or myopia. Risk of death or cancer is clearly a serious harm, but, in addition to physical harms, psychosocial harms need also to be considered and these may be more difficult to rank. In cases where the decision is made not to share genetic information, in addition to the harms associated with the condition itself, there are other harms such as those arising out of not having the possibility of making fully informed reproductive choices. Or perhaps, in those late onset conditions where there is no available intervention, the harms associated with not having been able to make more informed choices about other aspects of one's lifestyle.

Finally, to complicate matters further, where the decision is taken to disclose, there are the potential harms arising out of the desire not to have known. In any particular case, several of these types of harms may be present. Consider, for example, the following case study.

\section{Case 1}

Jim (60) has recently been diagnosed with Huntington's disease (HD), which has been confirmed molecularly. There is no reported family history of $\mathrm{HD}$, but, in retrospect, several of his deceased relatives may also have had the condition. Jim himself was initially diagnosed with Alzheimer's disease (AD) in his early 50s, but the diagnosis was reviewed following brain scans and revised to HD at the age of 57 years. Jim's capacity to consent is uncertain as he has marked dementia, but he has expressed a view, strongly shared with his wife Mary, that he does not want any of their four children (aged 27-37 years) to be informed of the diagnosis or of the risk to them. Jim and Mary can only see negative consequences arising out of the sharing of such information and anticipate that they will be blamed. Mary says; 'they either have it or don't'. The couple have been advised by genetic staff that their children might in fact want to know and may want to make their own choices. They have been offered help in communicating with their children, but have declined.

Recently, one of the daughters was referred to the genetics team to discuss her father's $\mathrm{AD}$, the early deaths of several of his relatives and the implications this might have for her. At a staff case discussion, most health professionals in the team feel that Jim and Mary's confidentiality should be respected. Justifications for this are centred around the lack of effective interventions for
HD. Nevertheless, concern is also expressed that the children have a right to know what they are actually at risk of.

\section{Magnitude of the risk}

A factor in addition to the seriousness of the harm itself will be the likelihood of its occurrence. Once again, genetics presents a wide spectrum of possibilities. In some conditions, such as HD, where the penetrance is very high, the risk of getting the disease is very high, or even certain if a certain Huntington gene repeat size is inherited. In others, the risk will be very low. In still others, the way the condition manifests will vary from person to person. In many cases, an assessment of the magnitude will not be at all straightforward. Consider the following case.

\section{Case 2}

Miss D, a 50-year-old woman is referred by her oncologist, because she has developed ovarian cancer. She has a strong family history of breast and ovarian cancer - several female relatives died of these diseases. She has other relatives with whom she has lost contact after a family rift some years ago. A diagnostic genetic test initiated by the oncologist reveals that Miss D has one of the many different possible BRCA1 mutations. When this result and its implications are explained to Miss D, she does not want anyone else in her family told that she has this mutation, or indeed to know that she has had cancer. She feels that she will be blamed for being the bearer of a faulty gene. It becomes apparent at a case discussion that, some months prior to this, Miss D's (unaffected) niece Mrs L had visited the same clinic and had requested a predictive genetic test for breast cancer; her mother had developed breast cancer at a young age and died from the disease. She was told that an effective predictive test (as contrasted with a diagnostic test) for breast/ovarian cancer requires the prior identification of a pathogenic mutation in an affected relative. Since she believed all her affected relatives to be dead and because despite clinical efforts no blood or tissue blocks were retrieved, she accepted that predictive genetic testing was not possible. Mrs L understands that she could be tested, but that a negative result would not be reassuring without knowing the familial mutation, because of the poor sensitivity of such testing. She wants a prophylactic mastectomy, and realises that only a predictive genetic test could demonstrate that this operation is not necessary.

When the genetics department discovers that the two women are related, they realise that they have, in Miss D's test results, the information they need to offer Mrs L an accurate predictive test, with a 50\% chance of her receiving 'good news'. Miss D has, however, refused permission for this to be divulged to other family members. At the case discussion, most of the team feel that Miss D's confidentiality is paramount. Though they do also feel that the 
benefits of disclosure to the wider family should be discussed with her at her next consultation. Nevertheless, as neither woman knows of the other's specific details, this in itself might prove difficult without breaking confidentiality. Some team members favour fudging the issue; 'Just look for that mutation in Mrs L. Miss D need not know'. But this is not a realistic option: Mrs L's understanding of genetics is good enough for this not to be possible without breaching confidentiality. Many team members feel that if she were to decide to have preventative surgery, disclosure would be justified without Miss D's consent, but would not consider the harm high enough if the disclosure was for 'knowledge only'. Some argued that this case is only a dilemma because the genetic test involved is still in its infancy. Once techniques improved, they suggest, Mrs L would be able to have an accurate test without the need to use Miss D's test result. This might turn out to be true, but the wider point remains. It seems likely that confirmation of a genetic diagnosis in one individual will continue to be necessary for accurate predictive testing in unaffected relatives. Without such prior confirmation, in many cases, it will not be clear which genetic mutation to look for.

\section{Avoidability of the harm and availability of 'interventions'}

The third element in any assessment of whether disclosure is justified is an assessment of to what extent the harm is 'avoidable'. What makes an intervention sufficiently morally significant to justify disclosure? Once again, genetics offers a wide range of possible 'interventions' following discovery of a genetic condition. While inherited conditions cannot usually be cured, they may be effectively treated (eg phenylketonuria) or their effects may be postponed (eg regular colonoscopies in familial polyposis). Effective medical interventions in BRCA1 families are less clear.

One might argue that little harm is done to Mrs $\mathrm{L}$ in not disclosing Miss D's test results, as it will simply mean that Mrs L will be unable to refine her risk. She already knows she is at increased risk and will be offered appropriate screening regimens anyway, which would not be altered if she were shown to have inherited the mutation. A counter argument to this position is provided by the fact that the available screening programmes (mammography, breast examination, ovarian ultrasound screening, etc) have limited evidence for their efficacy and have some - at least theoretical - risks (cumulative radiation, false positive and negative results, etc). There is a 50\% chance that Mrs L does not have the faulty gene and does not require such screening, and that she could be reassured that her risk of young onset breast/ovarian cancer is low. There is mounting evidence that prophylactic surgery does significantly reduce the risk of breast cancer, although it is a major operation with considerable morbidity attached to it. Respecting Miss D's confidentiality would mean that Mrs
L could not have a predictive test she might otherwise have. As a consequence, she may undergo prophylactic surgery unnecessarily. But it is not just the unnecessary hovering of the of the surgeon's knife that is an avoidable serious harm. She may simply continue to worry that she is at a high risk of young onset breast cancer, when in fact she is not.

Even in conditions, such as HD, where there are no effective medical treatments, other interventions or options are still possible. One of these is the possibility of informed reproductive choice and the use of techniques such as preimplantation genetic diagnosis or prenatal testing. Other possible 'interventions' might include more fully informed lifestyle choices of other kinds.

\section{Avoidability, risk and seriousness brought together}

These three elements, of seriousness, risk and availability of interventions, are clearly not independent of one another and the assessment of the justifiability of breaching confidentiality in any particular case will need to take into account the interplay between these factors. This will add increased complexity to any such assessment. Examples of situations in which this interplay will be morally significant and raise important ethical and practical difficulties will include the following:

- Cases in which there is a low risk of an extremely serious harm.

- Cases in which there is a high risk of less serious harms for which there is an effective intervention.

- Cases in which the harms are serious but the risks unclear or variable.

\section{Conclusion \\ Family communication is essential for a genetics service}

Good practice in clinical genetics naturally places a high value on working with families, in a way that is sensitive both to the care of the individual patient and to the fact that genetic information often has implications for other family members. This means that, in practice, genetic workers spend a lot of time developing good long-term relationships with patients and often with several members of the same family. In conversations with individual patients, a great deal of emphasis is placed on sensitive and supportive discussion of the advantages of informing other family members. This offers the best route to resolving most situations in which a clinician believes disclosure to be the right course of action. Even in families where disclosure is not actively refused, there is some evidence that appropriate disclosure does not always occur for a variety of different reasons such as distance of relationship, poor relationships or lack of openness about cancer. ${ }^{14}$ Some have argued for the use of a range 
of additional family-based mechanisms for reaching an agreement between family members, prior to testing on how information is to be shared. ${ }^{15-18}$ Nevertheless, while good clinical relationships, and perhaps family-based mechanisms of some kind, will often help patients to see the benefits of sharing information with relatives, this will not always be the case. There will continue to be cases where patients refuse to allow information to be shared and in which health professionals are faced with situations where they have to make decisions about whether or not to breach confidentiality. ${ }^{19,20}$ Although cases of the type we have described here may currently be thought to be relatively rare, a recent French study showed that nearly $10 \%$ of women attending a breast cancer genetic clinic would inform no one of any genetic testing result, and a third would inform one relative, but not necessarily all. ${ }^{10}$

In this paper, we have suggested that the relevant considerations will fall into three categories: seriousness of harm, size of risk and availability of interventions. The relative and overall strength of the relevant reasons will vary from case to case, depending on the nature of the condition and possible interventions, and in some cases on the intentions of the key actors. While it is clear that such decisions will need to be made on a case-by-case basis, we believe that it is vital that such decisions are based on sound and widely agreed principles. For this reason, we call for a broad and inclusive discussion about the question of under what kinds of conditions it is right to breach patient confidentiality and what ought to be the roles and responsibilities of individuals and family members in such decision making. As genetic medicine expands, many clinicians feel they have insufficient time or expertise for such debate in the routine clinical setting. ${ }^{21}$ We suggest that the time is ripe for dedicated forums for the debate of individual genetic cases with ethical problems. Ideally, these should have representation from a broad range of professionals. ${ }^{22}$ One possible model for such a forum is the recently formed UK Genethics Group, ${ }^{23}$ at which members of clinical genetics units meet several times a year, with the support of a medical ethicist and lawyer, to discuss dilemmas arising in the practice of clinical genetics.

\section{References}

1 Human Genetics Commission. Inside Information. Balancing interests in the use of personal genetic data; 1-5-2002.

2 Human Genetics: Choice and Responsibility. New York, NY: Oxford University Press; 1998.

3 Genetic Interest Group. Confidentiality guidelines, 1998; www.gig.org.uk (accessed 9/2002).

4 Knoppers B, Strom C, Wright-Clayton E et al. Professional disclosure of familial genetic information. Am J Hum Genet 1998; 62A: 474-483.

5 General Medical Council: Confidentiality: protecting providing information. Duties of a Doctor 2000.

6 W v Egdell, 1 All ER 855, 1990.

7 Tarasoff $\mathrm{v}$ Regents of University of CA, 551 P2d 334 (CA, 1976).

8 Pate v Threlkel, 661 So2d. 278 (FL, 1995) and Safer v Pack, 677 A. 2d 1188, 683 A 2d 1163 (NJ, 1996).

9 House of Commons Select Committee on Science and Technology: Human Genetics: The Science and its Consequences. Report No. 3 London: HMSO, 1995.

10 Julian-Reynier C, Eisinger F, Chabal F et al Disclosure to the family of breast/ovarian cancer genetic test results: patient's willingness associated factors. Am J Med Genet 94: 13.

11 Sommerville A, English V: Genetic privacy: orthodoxy or oxymoron? J Med Ethics 1999; 25: 144-150.

12 Knoppers BM, Hirtle M, Lormeau S, Laberge CM, Laflamme M: Control of DNA samples. Inform Genom 50: 385-401.

13 Nuffield Council on Bioethics. Genetic Screening, Ethical Issues, 1993; www.nuffieldbioethics.org/pubs.

14 Claes E, Evers-Kiebooms G, Boogaerts A, Decruyenaere M, Denayer L, Legius E: Communication with close and distant relatives in the context of genetic testing for hereditary breast and ovarian cancer in cancer patients. Am J Med Genet 2003; 116: $11-19$.

15 Weijer C: Benefit-sharing and other protections for communities in genetic research. Clin Genet 2000; 58: 367-368.

16 Leung WC, Mariman EC, van der Wouden JC, van Amergongen H, Weijer C: Results of genetic testing: when confidentiality conflicts with a duty to warn relatives. Br Med J 2000; 321: 1464-1466.

17 Doukas DJ: Autonomy beneficence in the family: describing the family covenant. J Clin Ethics 1991; 2: 145-148.

18 Doukas DJ, Berg JW: The family covenant genetic testing. Am J Bioethics 2001; 1: 2-10.

19 Knoppers BM: Genetic information and the family: are we our brother's keeper? Trends Biotechnol 2002; 20: 85-86.

20 Parker M, Lucassen A: Concern for families and individuals in clinical genetics. J Med Ethics 2003; 29: 70-73.

21 Lucassen AM, Houlston RS: Clinical geneticists' attitudes and practice towards testing for breast cancer susceptibility genes. $J$ Med Genet 2000; 37: 157-160.

22 Parker M, Lucassen A: Lancet 2002; 360: 1685-1688.

$23 \mathrm{http} / / /$ www.clingensoc.org/Ethics/genethics_club.htm (accessed 7/03). 